\title{
A Convenient Arithmetic for the Calculation of Radar System Errors Caused by Atmospheric Refraction
}

\author{
Gongke $\mathrm{Li}^{\mathrm{a}}$, Xiaoju Yong ${ }^{\mathrm{b}}$ \\ The Aviation Maintenance for NCO, Air Force Engineering University, Xinyang, 464000,China \\ aigongke2010@sina.com, byongxiaoju1987@126.com
}

Keywords: radar system errors; atmospheric refraction; segmented model; spherical stratification; least square; polynomial fitting

\begin{abstract}
Attribute to the problem that the existing radar system errors estimation algorithm is complex and the inherence relationship between the errors and the factors that caused the errors is hidden, a convenient arithmetic for radar system errors calculation is proposed in this paper. The main contributions of this paper are that, the less sensitive one is found and ignored among the factors, and an errors-function of these remained sensitive factors is obtained. Firstly, the spherical stratification method is introduced, and the errors are turn out to be affected by the range and elevation between target and radar, as well as the altitude of radar. Secondly, by a numerical simulation, the altitude is proved to be less sensitive to the errors. Lastly, an errors-function with respect to these much more sensitive factors is obtained by polynomial fitting. As a result, the computational cost is greatly reduced. The simulation results show that the proposed method can estimate the radar errors with high precision.
\end{abstract}

\section{Introduction}

The radar system errors estimation plays an important role in target navigation, target tracking and spatial registration[1]. An effective estimation method can greatly improve the precision of the applications to tracking and navigation. However, without radar system errors estimation, the data fusion and networked guidance will not reach their maximal efficiency[2]. So it appears to us that how to estimate the radar system errors is a fatal problem in many matters.

This paper concerns with the radar system errors caused by the refractive-index variations at microwave frequencies in the atmosphere. To our knowledge, there are many factors that influence the radar system error, such as fixed error of crystal. Due to the asymmetry of the atmospheric, the error introduced into measurements by refraction of the radar wave as it propagates through the atmosphere[4,5]. The major variation of refractive-index is that of a general decrease toward unity with altitude as the air density decreases. As a consequence, the radar beam promulgate through a curve instead of a line. That is the reason why the radar errors on range and elevation occur.

In recent years, the spherical stratification method is the common algorithm to analyses the influence of atmospheric refraction to the radar beam promulgation[6,7,8]. To utilize this method, we should get the model of atmospheric refraction index first. As we know, the development of the atmospheric refraction index model come through the rough to exact, and many models has been proposed such as linear model, exponential model, dual- exponential model, Hopfield model and so on[9-12]. Take computational complexity and precision into consideration, subsection model is utilized to modeling the atmospheric refraction in his paper. Based on the atmospheric refraction model, there are two main methods to analyze the influence of the atmospheric refraction to the promulgate way of radar electromagnetic wave. One is spherical stratification on the ground of the assumption that the atmospheric is symmetrical on plane[13]. The other is based on three-dimensional atmospheric method which considers 3d distribution of the atmosphere and with a high precision. However, the 3d distribution of the atmosphere is hardly to obtain at present[14]. Therefore, the first method is taken into account in this paper. However, no matter which method is chosen, the limitation of these two algorithms is that only a computational approach, calculating the radar systems errors on range and elevation according to the altitude of radar and target, the 
apparent range and apparent elevation, is given. As a consequence, the inherence connection of the outputs and inputs is not clear. Additionally, the calculating steps are complicated.

To solve this problem, a simple function respect to these variables above is obtained in this paper. Firstly, the segmented model is taken to modeling the atmospheric refraction-index. Secondly, the spherical stratification method is utilized to analyze the radar system error caused by the inhomogeneity of atmospheric. Thirdly, the sensitivity of the factors are compared by a numeric simulation, and the less sensitive factor is ignored. Furthermore, a filtering Errors-Function(EF) on these main factors is obtained by least square method. On the ground of the EF, the relationship between the altitude, the apparent range and apparent elevation and radar system errors is obvious. Moreover, the radar system error can be computed conveniently. So this function can be widely used in many applications.

\section{Models of Refraction Index}

To our knowledge, there are many ways to model the atmospheric refraction. Among these methods, the segmented model and Hopfield model are the common algorithms. Different model suits different area characteristic. Considering the atmospheric character of china, the segmented model [9] proposed by Bean and Thayer is chosen in this paper.

In their model, nature of refractivity is such that on the average it has a fairly linear altitude gradient to about $1 \mathrm{~km}$ above ground, then decays exponentially beyond that. Below an altitude of 9 $\mathrm{km}$, the refractivity depends on surface conditions, which varies with region, season, time of day, etc. Above $9 \mathrm{~km}$, the refractivity is relatively surface-condition independent. The segmented model is described as follow.

$$
N(h)= \begin{cases}N_{0}-G\left(h-h_{0}\right), & h_{0} \leq h \leq h_{0}+1 \\ N_{1} \exp \left(-c_{1}\left(h-h_{0}-1\right)\right), & h_{0}+1 \leq h \leq 9 \\ N_{9} \exp \left(-c_{9}(h-9)\right), & 9 \leq h \leq 60\end{cases}
$$

Where $h_{0}$ is the altitude of surface, the measurement of altitude is made in $\mathrm{Km}$. $G$ is the grads of the atmospheric refraction rate $1 \mathrm{Km}$ above the surface, the measurement of which is made in $\mathrm{N} / \mathrm{km} . N_{0}$ is a measure of refractivity in $\mathrm{N}$-units at the surface. $N_{1}$ and $N_{9}$ are the a measure of refractivity in $\mathrm{N}$-units at $1 \mathrm{Km}$ and $9 \mathrm{KM}$ above the surface, respectively. $c_{1}$ and $c_{9}$ are the attenuation index of the atmospheric-index with the altitude between $1 \mathrm{Km}$ to $9 \mathrm{Km}$ and higher than $9 \mathrm{Km}$, respectively, the measurements of which are made in $\mathrm{Km}^{-1}$.

\section{Radar System Error}

Spherical Stratification Method. In this section, the spherical stratification method is adopted to analyze the radar system error caused by atmosphere refraction. With the help of this algorithm, the influence of atmosphere asymmetry on the promulgate path of electromagnetic wave is studied.

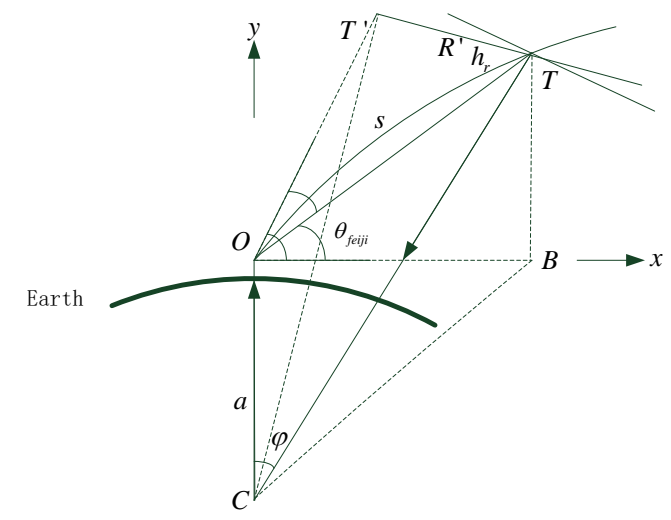

Fig.1 The geometry sketch map on the impact of atmosphere refraction on the promulgation of radar electromagnetic wave 
Firstly, the spherical stratification method is reviewed. As shown in Fig.1, $O$ and $T$ are the positions of radar and target, respectively. $s$ is the promulgation path of radar electromagnetic, $T^{\prime}$ is the apparent position of target, $L$ means the horizontal distance between radar and target. The promulgation of radar electromagnetic wave is not still a line, so the apparent range between target and radar is

$$
R^{\prime}=10^{-6} \int_{0}^{s} N d s=\int_{h_{-} \text {radar }}^{h_{T}} n \csc d h
$$

Where $s$ is the promulgate path. $N$ denotes the atmosphere refraction rate on the promulgate path. $n$ stands for refraction-index, and $n=1+N \times 10^{-6}$. $\theta$ is the elevation on the promulgate path.

Assume that the atmosphere is horizontally stratified, with the help Using Snell's law, the following can be obtained:

$$
\csc \theta=\frac{n(a+h)}{\sqrt{n^{2}(a+h)^{2}-n_{f e i j i}^{2}\left(a+h_{f e i j i}\right)^{2} \cos ^{2} \theta_{f e i j i}}}
$$

Where $h, n, \theta$ stand for the altitude, the refraction-index and the elevation on the promulgate path, respectively. $h_{f e i j i}, n_{f e i j i}$ and $\theta_{\text {feiji }}$ are said to be the altitude of radar, the refraction-index and the elevation of the target, respectively. $a$ is the radius of the earth.

Based on the geometric relation of target and radar

$$
\tan \theta_{\text {feiji }}=\frac{h_{T}-h_{\text {feiji }}}{L}
$$

Where $L$ means the horizontal distance between radar and target.

Based on Eq. (4), the apparent distance is

$$
\operatorname{Re}=\int_{h_{\text {feiji }}}^{h_{T}} \frac{n^{2}(a+h)}{\sqrt{n^{2}(a+h)^{2}-n_{\text {feiji }}^{2}\left(a+h_{\text {feiji }}\right)^{2} \cos ^{2} \theta_{\text {feiji }}}} d h
$$

As shown in Fig.1, the target geocentric field angle $\varphi$ is

$$
\varphi=\int_{h_{\text {feiji }}}^{h_{T}} \frac{\cot \theta}{a+h} d h=n_{\text {feiji }}\left(a+h_{\text {feiji }}\right) \cos \theta_{f e i j i} \times \int_{h_{\text {feiji }}}^{h_{T}} \frac{1}{(a+h) \sqrt{n^{2}(a+h)^{2}-n_{f e i j i}^{2}\left(a+h_{\text {feiji }}\right)^{2} \cos ^{2} \theta_{f e i j i}}} d h
$$

And the true elevation can be calculated according to the law of sines:

$$
\alpha_{\text {feiji }}=\arctan \left[\frac{\left(a+h_{T}\right) \cos \varphi-\left(a+h_{\text {feiji }}\right)}{\left(a+h_{T}\right) \sin \varphi}\right]
$$

Next, the true range can also be calculated according to the law of sines:

$$
R_{0}=\frac{\left(a+h_{T}\right) \sin \varphi}{\cos \alpha_{\text {feiji }}}
$$

Now, the radar system error on range and elevation caused by atmosphere refraction is

$$
\left\{\begin{array}{l}
\varepsilon=\theta_{0}-\alpha_{\text {feiji }} \\
\Delta r=R_{e}-R_{0}
\end{array}\right.
$$

Where $\varepsilon$ and $\Delta r$ are the errors on range and elevation, respectively.

Polynomial Fitting. From Eq.(2) to Eq.(9), we can find a fact that the errors are related to the radar altitude, target altitude, the distance and elevation between target and radar. In this section, we will try to get a function on these main items to calculate the system errors directly. Before the polynomial fitting, in order to simplify the function expression, we should find out the less sensitive variable and ignore it. Here, we will use numeric simulations to compare impacts of these variables on the errors. Then, we will try to get the EF of these main items to calculate the system errors. 
Under the assumption that the range between target and radar change from $50 \mathrm{Km}$ to $400 \mathrm{Km}$, the altitude of radar change from $0.5 \mathrm{Km}$ to $6 \mathrm{Km}$, the elevation between target and radar change from $5^{\circ}$ to $50^{\circ}$, the errors on range and elevation are shown in Fig.2 and Fig.3.

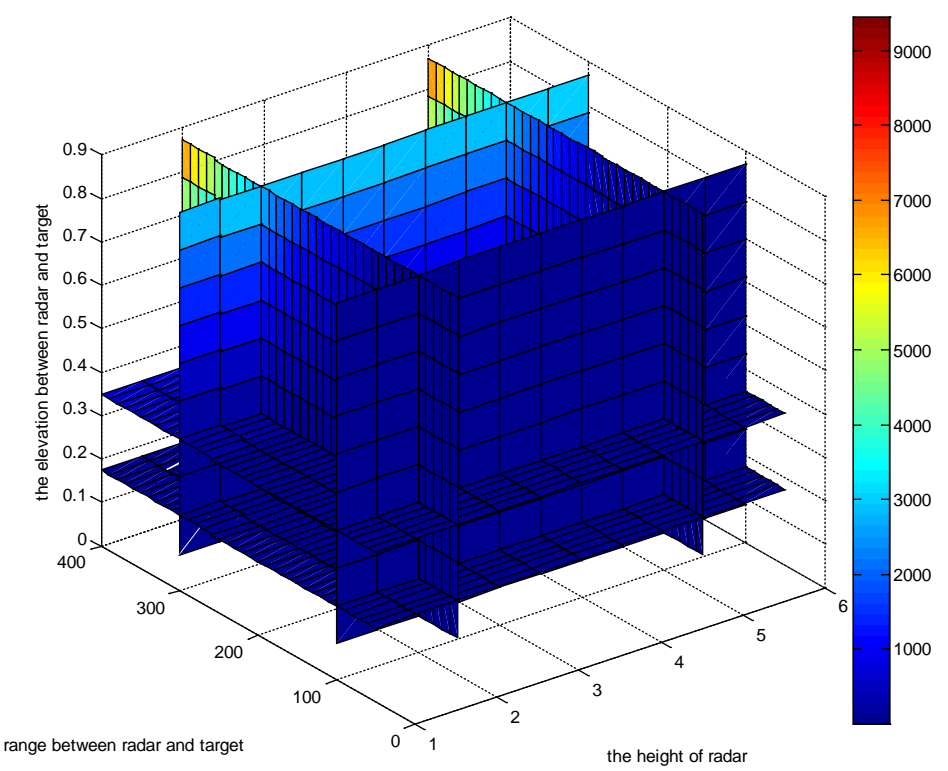

Fig. 2 The radar system error on range

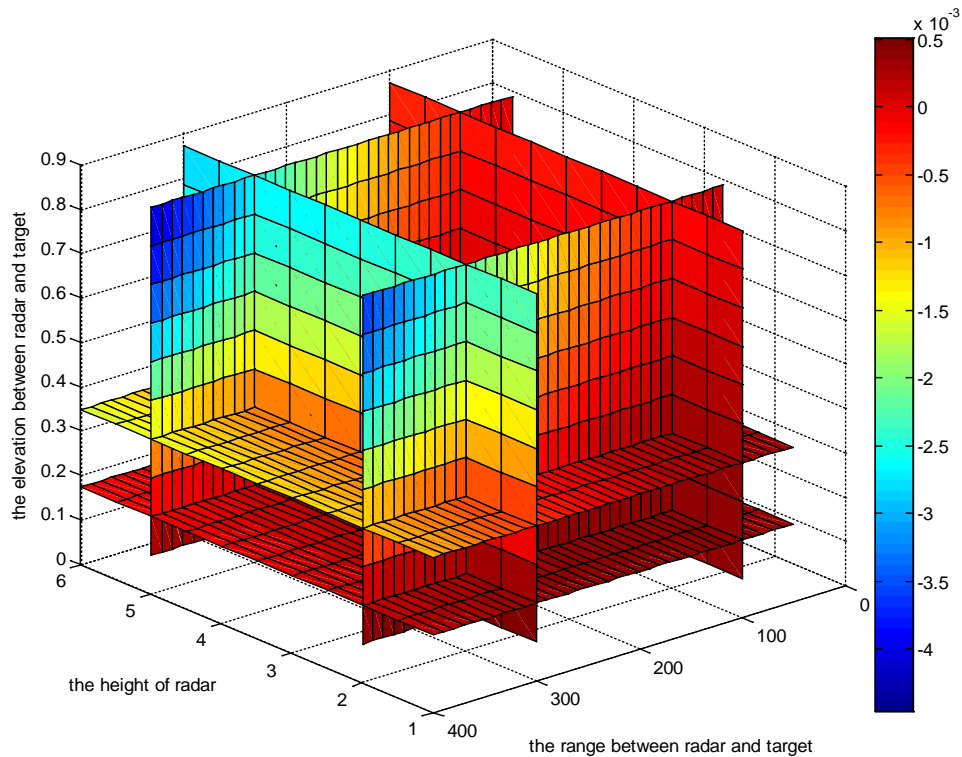

Fig. 3 The radar system error on elevation

Fortunately, we can find a factor that is not sensitive to the errors. Pay attention to the color which respects the radar system errors, as the altitude of radar change from $1 \mathrm{~km}$ to $6 \mathrm{~km}$, if the range and elevation are changeless, the color almost keeps the same which means that the radar system errors keep changeless. So we can draw a conclusion that the radar system errors are not sensitive to the altitude of radar. As contrary, the errors are sensitive to the elevation and range between radar and target.

The radar system errors are impacted by the range and elevation between radar and target and the altitude of radar, but from the analysis above, the main factors are the range and elevation. As a consequence, in what follows, we only consider the range and elevation between target and radar.

Adopting a quadric multinomial function, the form of the expression is as follow:

$$
\begin{gathered}
\Delta R=f(R, \theta) \\
\Delta \theta=g(R, \theta) \\
\Delta R=a_{11}+a_{12} \theta+a_{13} \theta^{2}+a_{14} R+a_{15} R \theta+a_{16} R \theta^{2}+a_{17} R^{2}+a_{18} R^{2} \theta+a_{19} R^{2} \theta^{2} \\
\Delta \theta=a_{21}+a_{22} \theta+a_{23} \theta^{2}+a_{24} R+a_{25} R \theta+a_{26} R \theta^{2}+a_{27} R^{2}+a_{28} R^{2} \theta+a_{29} R^{2} \theta^{2}
\end{gathered}
$$


The least square rule is taken to fitting the EF, the object function is

$$
\min \mathbf{E}=\sum_{i=1}^{m} \sum_{j=1}^{n}\left(\mathbf{z}_{R}(i, j)-\Delta \mathbf{R}(i, j)\right)^{2}
$$

And

$$
\min \mathbf{E}=\sum_{i=1}^{m} \sum_{j=1}^{n}\left(\mathbf{z}_{\theta}(i, j)-\Delta \boldsymbol{\theta}(i, j)\right)^{2}
$$

Where $(m, n)$ is the size of $\mathbf{R}, \mathbf{z}_{R}$ and $\mathbf{z}_{\theta}$ are the radar system errors acquired from Eq.(2) to Eq.(9), respectively. The range, elevation and altitude are measured by $\mathrm{Km}$, mrad and $\mathrm{Km}$, respectively. The radar system errors on range and elevation are measured by $\mathrm{m}$ and mrad, respectively.

Here, we take the function $\Delta R$ for example to solve the problem. The coefficient vector $\mathbf{a}=\left[\begin{array}{lllllllll}a_{11} & a_{12} & a_{13} & a_{14} & a_{15} & a_{16} & a_{17} & a_{18} & a_{19}\end{array}\right]^{T}$ should satisfy

$$
\frac{\partial \mathbf{E}}{\partial a_{1 i}}=\mathbf{0}
$$

Substitute Eq.(11), Eq.(12) to Eq.(14), then

Where

$$
\mathbf{H a}=\mathbf{Z}
$$

$$
\begin{aligned}
& {\left[\begin{array}{lllllllll}
\varphi(0,0) & \varphi(0,1) & \varphi(0,2) & \varphi(1,0) & \varphi(1,1) & \varphi(1,2) & \varphi(2,0) & \varphi(2,1) & \varphi(2,2)
\end{array}\right.} \\
& \varphi(0,1) \quad \varphi(0,2) \quad \varphi(0,3) \quad \varphi(1,1) \quad \varphi(1,2) \quad \varphi(1,3) \quad \varphi(2,1) \quad \varphi(2,2) \quad \varphi(2,3) \\
& \varphi(0,2) \quad \varphi(0,3) \quad \varphi(0,4) \quad \varphi(1,2) \quad \varphi(1,3) \quad \varphi(1,4) \quad \varphi(2,2) \quad \varphi(2,3) \quad \varphi(2,4) \\
& \varphi(1,0) \quad \varphi(1,1) \quad \varphi(1,2) \quad \varphi(2,0) \quad \varphi(2,1) \quad \varphi(2,2) \quad \varphi(3,0) \quad \varphi(3,1) \quad \varphi(3,2) \\
& \mathrm{H}=\varphi(1,1) \quad \varphi(1,2) \quad \varphi(1,3) \quad \varphi(2,1) \quad \varphi(2,2) \quad \varphi(2,3) \quad \varphi(3,1) \quad \varphi(3,2) \quad \varphi(3,3) \\
& \varphi(1,2) \quad \varphi(1,3) \quad \varphi(1,4) \quad \varphi(2,2) \quad \varphi(2,3) \quad \varphi(2,4) \quad \varphi(3,2) \quad \varphi(3,3) \quad \varphi(3,4) \\
& \varphi(2,0) \quad \varphi(2,1) \quad \varphi(2,2) \quad \varphi(3,0) \quad \varphi(3,1) \quad \varphi(3,2) \quad \varphi(4,0) \quad \varphi(4,1) \quad \varphi(4,2) \\
& \varphi(2,1) \quad \varphi(2,2) \quad \varphi(2,3) \quad \varphi(3,1) \quad \varphi(3,2) \quad \varphi(3,3) \quad \varphi(4,1) \quad \varphi(4,2) \quad \varphi(4,3) \\
& {\left[\begin{array}{lllllllll}
\varphi(2,2) & \varphi(2,3) & \varphi(2,4) & \varphi(3,2) & \varphi(3,3) & \varphi(3,4) & \varphi(4,2) & \varphi(4,3) & \varphi(4,4)
\end{array}\right]}
\end{aligned}
$$

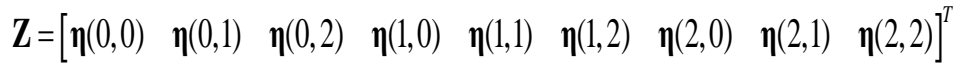

$$
\begin{aligned}
& \boldsymbol{\varphi}(p, q)=\sum_{i=1}^{m} \sum_{j=1}^{n} \mathbf{R}^{p}(i, j) \boldsymbol{\theta}^{q}(i, j) \text {, and } \boldsymbol{\eta}(p, q)=\sum_{i=1}^{m} \sum_{j=1}^{n} \mathbf{z}_{R}(i, j) \varphi(p, q) \text {. }
\end{aligned}
$$

So, the coefficient vector $\mathbf{a}$ is

$$
\mathbf{a}=\mathbf{Z} / \mathbf{H}
$$

Using the data in the simulation above, the coefficients of the EF could be obtained, which are shown as follow:

$$
\begin{gathered}
a_{11}=138.3313, a_{12}=-0.1370, a_{13}=3.5907 \times 10^{-5}, a_{14}=-1.5251, a_{15}=0.0017, a_{16}=-5.2007 \times 10^{-7}, \\
a_{17}=0.0049, a_{18}=-5.8185 \times 10^{-6}, a_{19}=2.3228 \times 10^{-9} .
\end{gathered}
$$

Adopt the method above similarly, the coefficients of the function $\Delta \boldsymbol{\theta}$ could be obtained, which are shown as follow:

$$
\begin{gathered}
a_{21}=-31.5830, a_{22}=0.0344, a_{23}=-5.7131 \times 10^{-6}, a_{24}=0.4947, a_{25}=-4.2515 \times 10^{-4}, \\
a_{26}=6.9947 \times 10^{-8}, a_{27}=-0.0012, a_{28}=9.4424 \times 10^{-7}, a_{29}=-2.0144 \times 10^{-10} .
\end{gathered}
$$

Now, we can get a EF to calculate the radar system errors directly and expediently. 


\section{Simulations}

In this section, the validity of the atmospheric refraction index model and newly got function will be checked out.

The Atmospheric Refraction Index Model. Based on the model in section 2, using the parameters in [16]. The atmospheric refraction index model is shown in Fig.4.

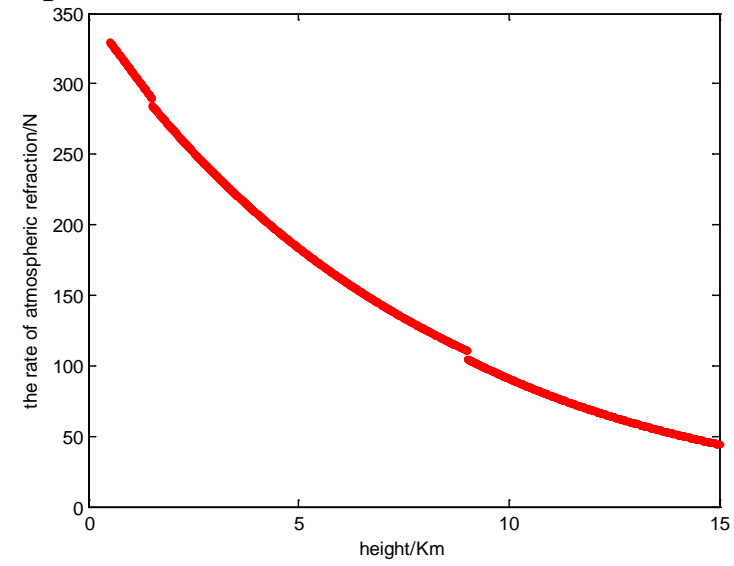

Fig. 4 The atmospheric refraction index model based on the segmented model

From Fig.4, we can find that the atmospheric refraction rate decrees and the slope gradually lessen with the decrees of the altitude. When the altitude is $15 \mathrm{Km}$, the atmospheric refraction rate is $49 \mathrm{~N}$.

The Polynomial Fitting Function. In this section, the validity of the function in section 3.2 will be checked out. Assume that the altitude is $6 \mathrm{Km}$, the distance between radar and target is $50 \mathrm{Km}$ to $400 \mathrm{Km}$, the elevation between target and radar is $5^{\circ}$ to $50^{\circ}$, the radar system errors calculated based on Eq.(2) to Eq.(9) are shown in Fig. 5 and Fig. 8. The radar system errors calculated related EF are shown in Fig.6 and Fig.9. The precision of the EF is disrupted by a corresponding variable $\boldsymbol{\alpha}$, which is defined as

$$
\boldsymbol{\alpha}(i, j)=\frac{\mathbf{e}(i, j)}{\mathbf{Z}(i, j)}=\frac{\mathbf{f}(i, j)-\mathbf{Z}(i, j)}{\mathbf{Z}(i, j)}
$$

Where $\mathbf{f}(i, j)$ is the error computed based on the EF, and $\mathbf{Z}(i, j)$ is the error computed corroding to the spherical stratification method. And the results are shown in Fig.7 and Fig. 10.

Compare Fig. 5 and Fig. 8 with Fig. 6 and Fig. 9, with the help of $\boldsymbol{\alpha}$, we can draw the conclusion that the EF proposed in this paper can exactly descript the errors caused by the atmospheric refraction. 


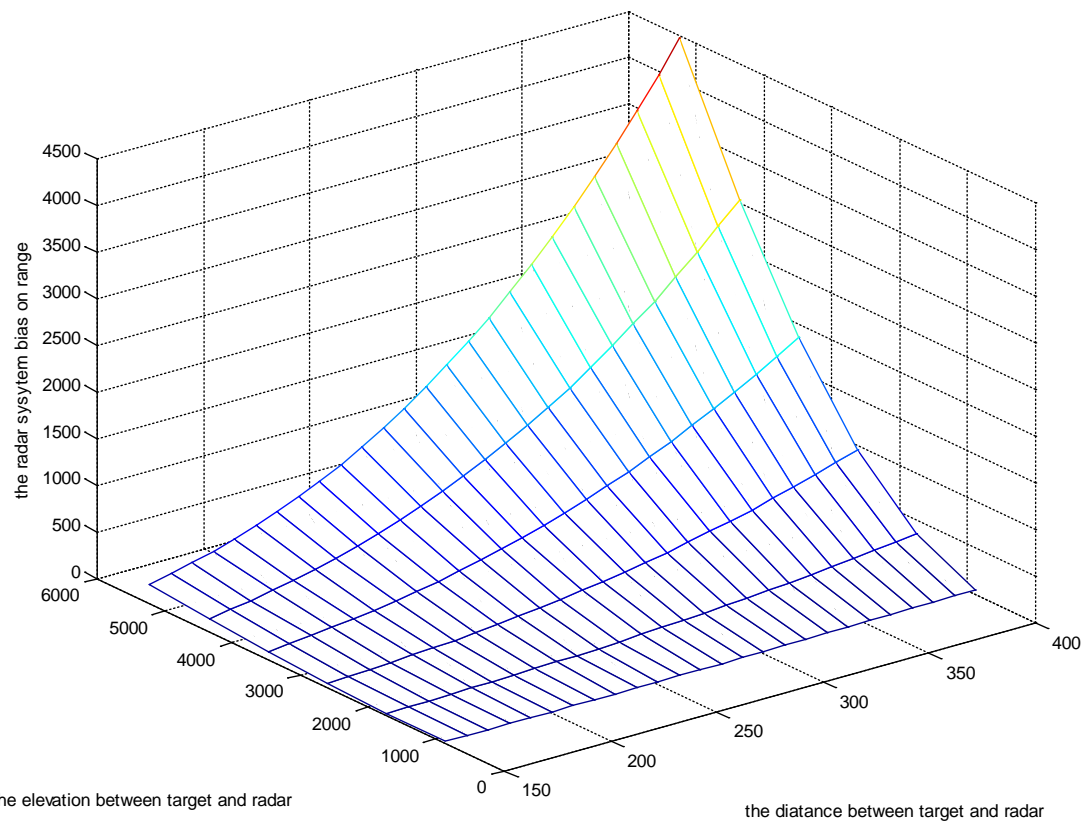

Fig. 5 The radar system error on range calculate from Eq.(2) to Eq.(9)

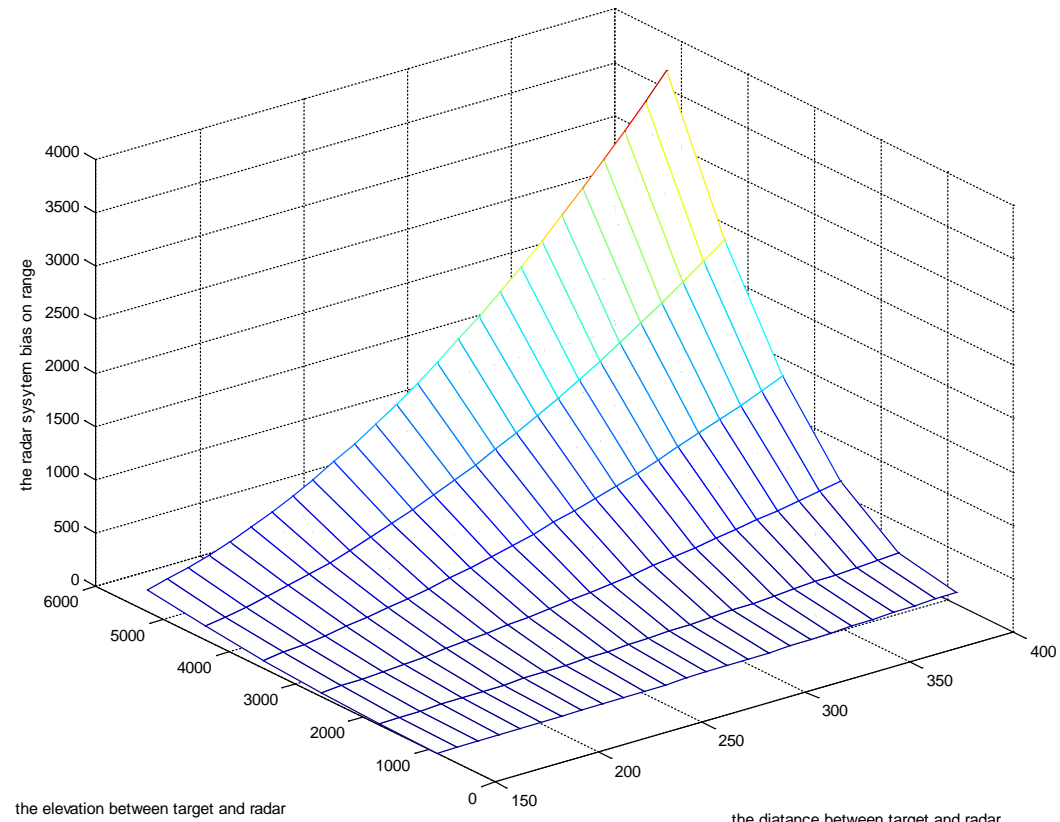

Fig. 6 The radar system error on range calculated based on the EF in this paper

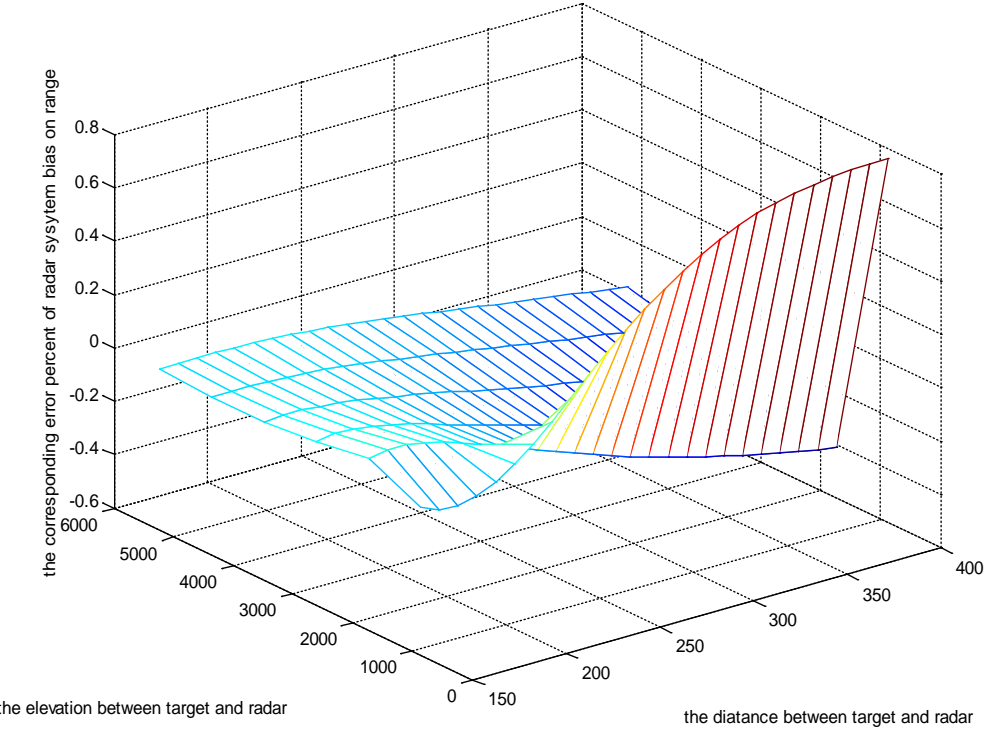

Fig. 7 The corresponding $\boldsymbol{\alpha}$ on range 


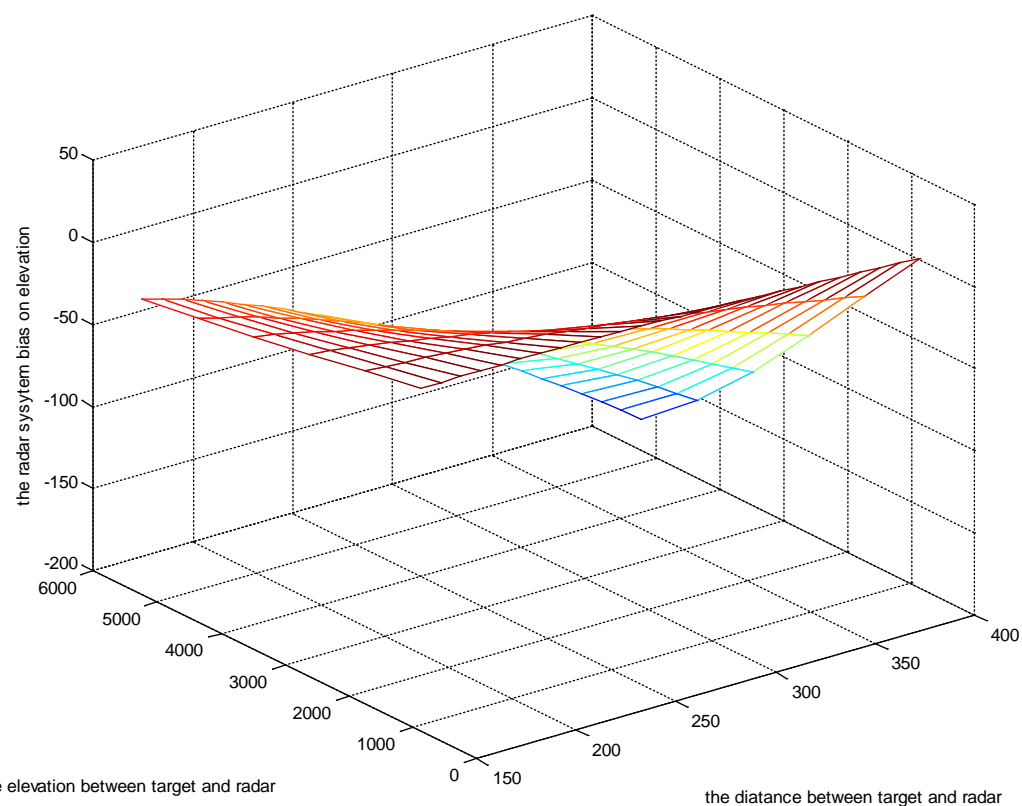

Fig. 8 The radar system error on elevation calculate from Eq.(2) to Eq.(9)

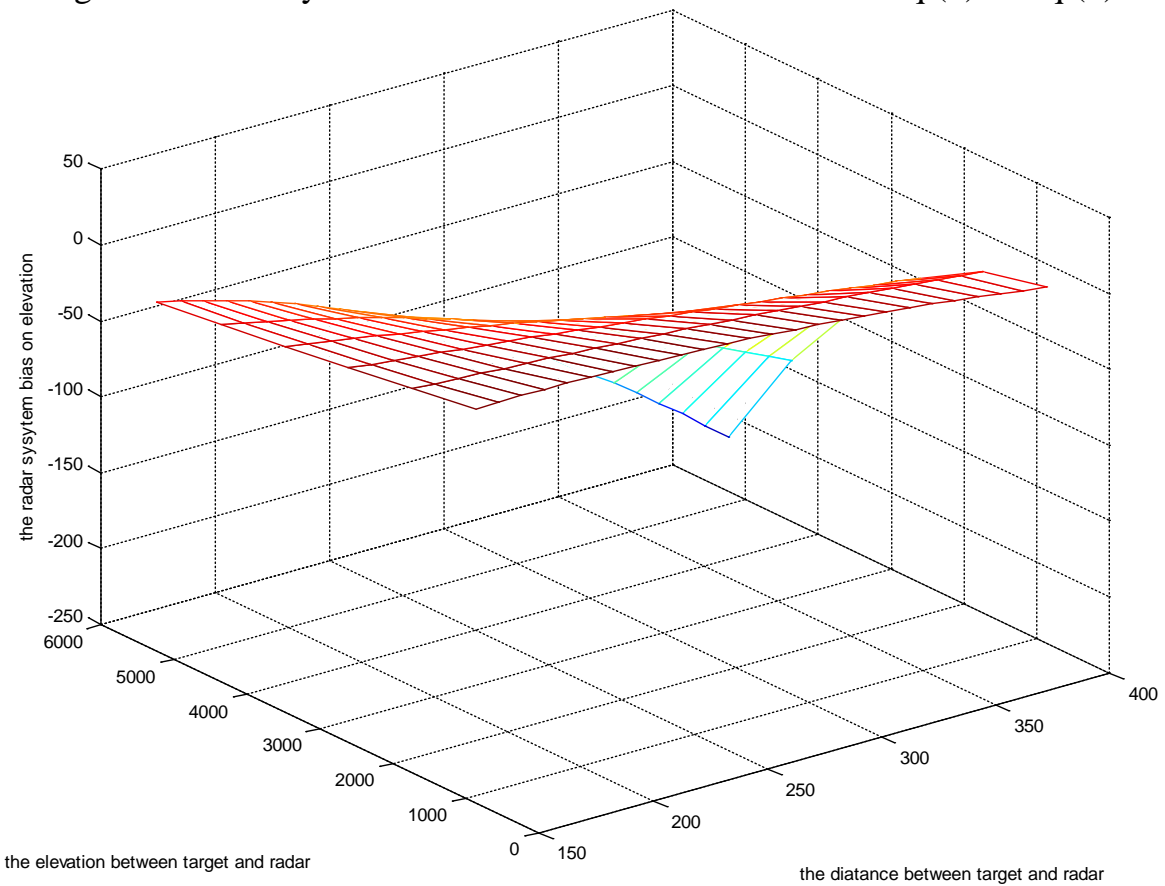

Fig. 9 The radar system error on elevation calculated based on the EF in this paper 


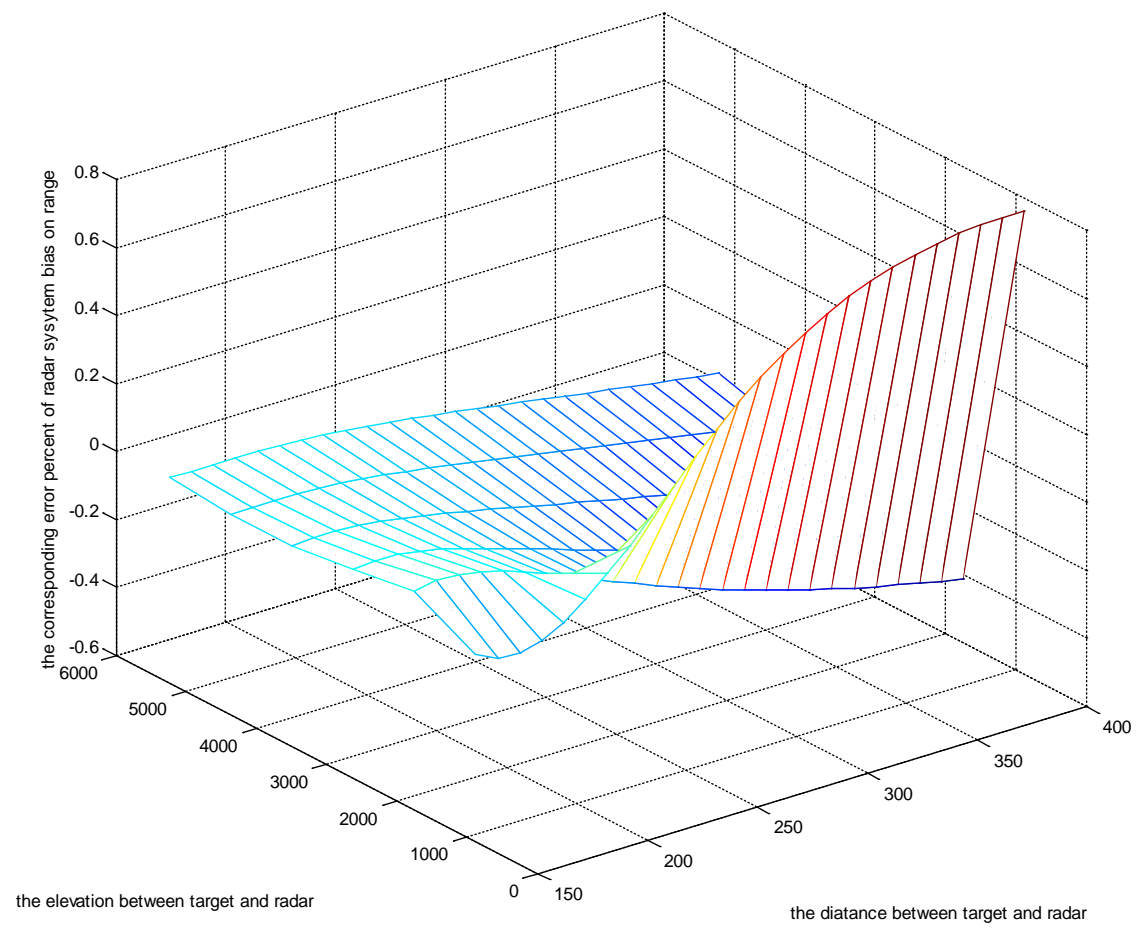

Fig. 10 The corresponding $\boldsymbol{\alpha}$ on elevation

\section{Conclusion}

In this paper, the radar system errors caused by atmospheric refraction are analyzed. To predigest the spherical stratification method, by comparing the main factors influenced on the system errors such as altitude, distance and elevation, the more sensitive variables are chosen. Then a system errors function of these more sensitive factors is drawn based on the polynomial fitting method. The limitation of this paper is that the radar system errors function is only related to the atmospheric refraction, and omitting other units.

\section{References}

[1] J.C. Kerce, W.D. Blair, G..C. Brown. Modeling Refraction Errors for Simulation Studies of Multisensor Target Tracking.

[2] A K Shukla, D J Fraser, A Lockton, A H Y Chan, J E N Field, S L Shemar. The Impact of Tropospheric Propagation on Data Fusion from Multiple Radars. 2005 7th International Conference on Information Fusion.

[3] Barton, David K., and Ward, Harold R., "Handbook of Radar Measurement,” Artech House, Inc., Dedham MA, 1984, Appendix D.

[4] G. H. Millman. Atmospheric effects in VHF and UHF propagation. Proceedings ofthe IRE, vol. 46, pp. 1492-1501, August 1958.

[5] W. L. Andersont, N. J. Beyers, R. J. Rainey. Comparison of Experimental with Computed Tropospheric Refraction. IRE Transactions on Antennas and propagation. 1960,9.

[6] A. W. Doerry. " Correcting radar range measurements for atmospheric propagation effects ", Proc. SPIE 9077, Radar Sensor Technology XVIII, 90771K (May 29, 2014).

[7] Iannini, L.; Dipt. di Elettron., Politec. di Milano; Atmospheric Phase Screen in Ground-Based Radar: Statistics and Compensation. Geoscience and Remote Sensing Letters, IEEE, 2011, Vol.8, No.3:537 - 541.

[8] Armin W. Doerry, "Depression and Grazing Angles with Earth Curvature and Refraction”, 
Sandia Internal Memorandum, SAND99-2241, September 1999.

[9] B. R. Bean, G. D. Thayer, "Models of the Atmospheric Radio Refractive Index”, Proceedings of the I.R.E., Vol. 45, Issue 5, pp. 740-755, May 1959.

[10] Earnest K. Smith, Jr., Stanley Weintraub, “The Constants in the Equation for Atmospheric Refractive Index at Radio Frequencies”, Proceedings of the I.R.E., Vol. 4, Issue 8, pp. 1035-1037, August 1953.

[11] Armin W. Doerry. Earth Curvature and Atmospheric Refraction Effects on Radar Signal Propagation. SANDIA REPORT. 2013.01.

[12] Mareus, P., Nico, G., Tome, R. Experimental study on the atmospheric delay based on GPS, ASR Interferometry, and Numerical Weather Model Date. Geoscience and remote sensing, IEEE trans on. Vol51,no.1, 2013:6-11.

[13] Fred M. Dickey, Armin W. Doerry, Louis A. Romero, "Degrading effects of the lower atmosphere on long range airborne SAR imaging”, IET Proceedings on Radar, Sonar \& Navigation, Vol. 1, No. 5, pp. 329-339, October 2007.

[14] Edward E. Altshuler, "Tropospheric Range-Error Corrections for the Global Positioning System”, IEEE Transactions on Antennas and Propagation, Vol. 46, No 5, pp. 643-649, May 1998.

[15] Fan Zhang, Guojun Li, Wei Li, and Wei Hu. Multiband Microwave Imaging Analysis of Ionosphere and Troposphere Refraction for Spaceborne SAR. International Journal of Antennas and Propagation. 2014,1.

[16] GJB/Z87-97, Handbook of Refraction and Attenuation of Radiowave Propagation for Radar, People’s Republic of China National Military Standard, 1997.

[17] K. H. Craig. Clear air characteristics of the troposphere. In M. P. M. Hall, L. Barclay and M.T. Hewitt, editors, Propagation ofRadiowaves, pages 105-130, IEE, London, 1996.

[18] Anthony R. Lowry, Chris Rocken, Sergey V. Sokolovskiy and Kenneth D. Anderson. Vertical profiling of atmospheric refractivity from ground-based GPS. Radio Sci., 37(3): 13-1 - 13-18, 2002. 